\title{
Study on the Saturation Properties of Silicone Oil-Based Magnetorheological Fluids in Mechanical Engineering
}

\author{
Dongdong Wang ${ }^{1,2}$, Xinhua Liu ${ }^{*, 2,3}$, Lifeng Wang ${ }^{2}$, Yankun Ren ${ }^{2}$ and Qiuxiang Zhang ${ }^{2}$ \\ ${ }^{1}$ Xuyi Mine Equipment and Materials R\&D Center, China University of Mining \& Technology, Huai'an 211700, China \\ ${ }^{2}$ School of Mechatronic Engineering, China University of Mining \& Technology, Xuzhou 221116, China \\ ${ }^{3}$ The State Key Laboratory of Fluid Power Transmission and Control, Zhejiang University, Hangzhou310027, China
}

\begin{abstract}
In this paper some saturation properties of silicone oil-based magnetorheological fluids in mechanical engineering were researched and discussed by theoretical analysis and experiments. Firstly, experiment materials and preparation process of silicone oil-based magnetorheological fluids were presented. Secondly, magnetic-field saturation, particles saturation and added nano-particles saturation were elaborated. Finally, the influence of these properties on magnetorheological fluids properties were discussed to provide references for parameter design of magnetorheological fluids preparation and mechanical engineering.
\end{abstract}

Keywords: Added nano-particles, magnetic field, magnetic particles, saturation properties, silicone oil-based magnetorheological fluids.

\section{INTRODUCTION}

Magnetorheological fluids (MRFs) are kinds of typical intelligent materials that their rheological properties, such as the apparent viscosity, stiffness and yield stress, can be altered by an applied magnetic field. Due to its unique characteristics, MRFs behave as smart or functional fluids and have been more and more used in a variety of fields such as mechanical engineering, aerospace, precision processing engineering and control engineering [1-3]. The most important properties of MRFs are mechanical property and stability performance, but many influence factors would affect the properties such as applied magnetic-field, magnetic particles' properties, added nano-particles, etc. Since the first discovery of MRFs, there have been a large number of studies on MRFs characteristics and the influence factors [4].

By the means of Monte Carlo method, Masayuki Aoshima found that as the magnetic interaction between particles increases, many small clusters such as anti-parallel particle pairs, raft-like clusters, and small loop-like clusters were formed. These small clusters gathered to form larger aggregates [5]. Lattice-Boltzmann model was built by Xuan Yimin and the phenomena of flocculation process at zero fields and chainlike structure along field flux under extra uniform field was researched with this model [6]. In order to solve sedimentation problem, some academics added submicron nanosized fillers into MRFs and the effects of substitution of micron size powder by nanometer size powder in MRFs were investigated [7]. The relationships

*Address correspondence to this author at the School of Mechanical and Electrical Engineering, China University of Mining \& Technology, Xuzhou, 221116, P.R. China; Tel: +86-0516-83884512;

E-mail:1_xinhua_2006@126.com between MRFs yield stress and applied magnetic field strength were researched by Ginder through the finite element when the magnetic particles reached magnetic-field saturation [8]. The function relationship between MRFs yield stress and applied magnetic field intensity by experiment was researched by Felt [9]. MRFs with high permeability magnetic particles were researched by Bossis and the positive relationship between yield stress and MRFs volume fraction was investigated [10]. The phenomenon was found by Lemaire that the yield stress increases with the increase of particle diameter when the particle diameter was quite small and yield stress had nothing to do with particle size when the particle diameter was quite big [11]. The lord magnetic field of MRF and its circuit design are mainly expounded [12]. The influence of particles diameter on static yield stress was researched by Jiang and they found that static yield stress increased with the increase of particle diameter [13].

\section{EXPERIMENTAL METHODS}

MRFs materials are normally composed of magnetic particles and additives dispersed in a carrier liquid. In the experiment, soft magnetic carbonyl iron particles (density of carbonyl iron particles: $7.8 \mathrm{~g} / \mathrm{cm}^{3}$, average size of carbonyl iron particles: $3 \mu \mathrm{m}$ ) and nanometer $\mathrm{Fe}_{3} \mathrm{O}_{4}$ particles acquired from Beijing Dk nano technology Co. with a size of $20 \mathrm{~nm}$ are used as a dispersed phase. Dimethyl silicone oil (dynamic viscosity of Dimethyl silicone oil: $500 \mathrm{cSt}$ in $25^{\circ} \mathrm{C}$, density: $0.97 \mathrm{~g} / \mathrm{cm} 3$, Shin-Etsu, Japan) is used as suspending medium for MRFs. To improve the sedimentation stability and reduce aggregation, sodium dodecyl benzene sulfonate and oleic acid supplied are used as surfactant; diatomite powders and graphite are used as inorganic thixotropic agent and antiwear agent. Absolute ethyl alcohol is also used in the whole process of the experiment. 
In this paper the details of MRFs preparation are carried out as following process: Firstly, soft magnetic carbonyl iron particles, oleic acid and sodium dodecyl benzene sulfonate are mixed. Then the mixture is dispersed into a beaker containing absolute ethyl alcohol and underwent continuous stirring with a mechanical stirrer until the mixture is uniformly dispersed, thus soft magnetic carbonyl iron particles are coated sufficiently by surfactant. Secondly, the mixture is dispersed by an ultrasonic dispersion instrument in order to remove part of the aggregated grains. Thirdly, after this reaction, the reactants are milled by a planetary ball mill for $10 \mathrm{~h}$. Fourthly, the magnetic particles are separated from the obtained mixture by a magnet, and then they are washed by absolute ethyl alcohol for several times. After washing, the particles are dried in a vacuum oven at 75 degrees centigrade for $8 \mathrm{~h}$ under reduced pressure, and then grinding and sieving them. Finally, the modified particles, graphite and diatomite powders, dimethyl silicone oil and nanometer $\mathrm{Fe} 3 \mathrm{O} 4$ particles are mixed with $20 \%$ volume fractions of magnetic particles. Stirring rapidly and dispersing the resulted solution again.

In the experiment some parameters (current, magnetic field intensity, shear yield stress) of the MRFs we prepared are needed to be measured. The DC power which provides the different magnetic field is used to provide exciting currents for the experiment and relevant parameters listed in Table 1. The magnetic field intensity is measured by gaussmeter and relevant parameters listed in Table $\mathbf{2}$.

Shear yield stress of MRFs is tested by our self-designed experiment equipment (Rotating disk type magnetic rheology liquid yield stress tester) to research the shear yield performance under the action of magnetic field, as shown in Fig. (1), in which two parallel disks are used as coupling material.
Table 1. Main technical parameters of DC power.

\begin{tabular}{|c|c|c|c|}
\hline $\begin{array}{c}\text { Input } \\
\text { Voltage }\end{array}$ & $\begin{array}{c}\text { Outout } \\
\text { Current }\end{array}$ & $\begin{array}{c}\text { Voltage } \\
\text { Stability }\end{array}$ & $\begin{array}{c}\text { Current } \\
\text { Stability }\end{array}$ \\
\hline \hline $220 \mathrm{~V}$ & $0 \sim 10 \mathrm{~A}$ & $<0.01 \%+2 \mathrm{mV}$ & $<0.2 \%+2 \mathrm{~mA}$ \\
\hline
\end{tabular}

Table 2. Main technical parameters of gaussmeter.

\begin{tabular}{|c|c|c|c|}
\hline Frequency & $\begin{array}{c}\text { Measuring } \\
\text { Range }\end{array}$ & $\begin{array}{c}\text { Resolution } \\
\text { Ratio }\end{array}$ & $\begin{array}{c}\text { Communication } \\
\text { Interface }\end{array}$ \\
\hline \hline $50 \sim 60 \mathrm{~Hz}$ & $0 \sim 3 \mathrm{~T}$ & $0.01 \mathrm{mT}$ & RS-232 \\
\hline
\end{tabular}

The MRF samples are injected into the liquid storage and the electric current is adjusted to achieve different magnetic circuit. Shear yield stress of MRFs can be calculated by the reading of experiment equipment.

\section{RESULTS AND DISCUSSION}

\subsection{Saturation Property of Magnetic Field}

The magnetic force of MRFs between magnetic particles is usually calculated by magnetic dipole model. The model can be used to calculate the shear stress accurately only in relatively weak magnetic field, because the interaction effects between magnetic dipoles and saturation magnetization phenomenon are not taken into consideration. According to Frohlich-Kennelly formula, the magnetization intensity can be expressed as follows:

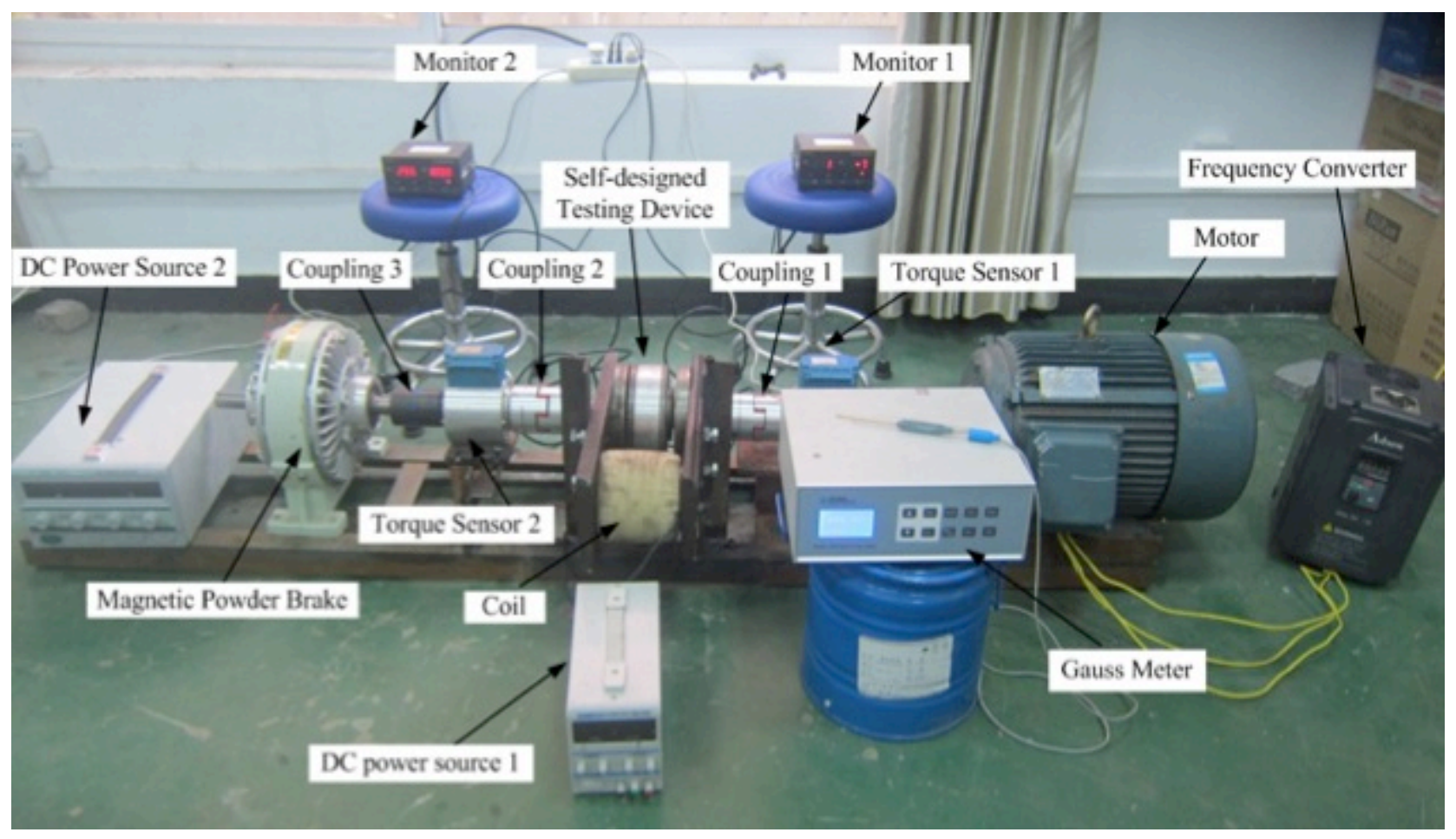

Fig. (1). Experiment equipment. 
$M=\frac{\chi^{0} H_{\mathrm{m}}}{1+\chi^{0} H_{m} / M_{s}}$

According to Ohm law in magnetic circuit, the equations of magnetic field intensity can be shown as follows:

$H l=H_{m}\left(l-l_{p}\right)+H_{p} l_{p}$

$M+H_{m}=H_{p}$

So the relationship between magnetization intensity and magnetic field intensity can be obtained as follows:

$\left[k+\frac{M_{s}}{\chi^{0}\left(M_{s}-M\right)}\right] M=H$

The magnetization process can be divided into two stages: linear process and saturation process. In the liner process the yield stress is liner changed with magnetic field. In the saturation process, the magnetization intensity of particles reaches saturation magnetization under the magnetic field intensity which is called critical magnetic field intensity. Two stages of the magnetization process can be expressed as follows:

$M=\left\{\begin{array}{cc}\frac{\chi^{0}}{1+\chi^{0} k} H, & H \leq H_{c} \\ M_{s} & H>H_{c}\end{array}\right.$

where $\mathrm{M}$ is the magnetization intensity, $\chi^{0}$ is the initial magnetic susceptibility of magnetic particles, $H_{\mathrm{m}}$ is the inner magnetic field intensity of magnetic particles and $M_{\mathrm{s}}$ is saturation magnetization intensity.

Three kinds of silicone oil-based MRFs which have different volume fractions of magnetic particles $10 \%, 20 \%$ and $30 \%$ have been prepared to research the phenomenon of magnetic-field saturation. Fig. (2) shows that the magnetic field intensity is changing with the field current of three tested MRFs. At the first stage $(\mathrm{H}<\mathrm{Hc})$, the magnetic field intensity exhibits approximately linear magnetic properties up to $2.5 \mathrm{~A}$ of applied field intensity. The magnetic flux

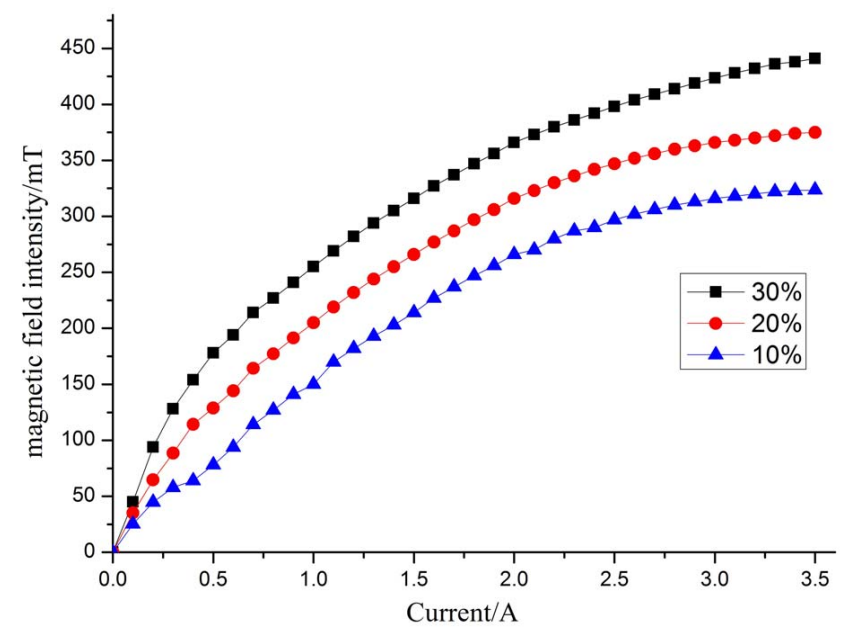

Fig. (2). Saturation phenomenon of magnetic-field. density of $30 \%$ fractions demonstrates greater values over those of the other MRFs because of a higher particle density. The phenomenon of magnetic-field saturation occurs with the increase of applied magnetic field. At this stage $(\mathrm{H}>\mathrm{Hc})$, the growth rate of magnetic field intensity slows down and tends to a certain value at last.

\subsection{Saturation Property of Magnetic Particles}

The magnetic particles are one of the most important parameter which affects the MRFs properties. Volume fractions of magnetic particles and magnetic particle diameter are the most important factors.

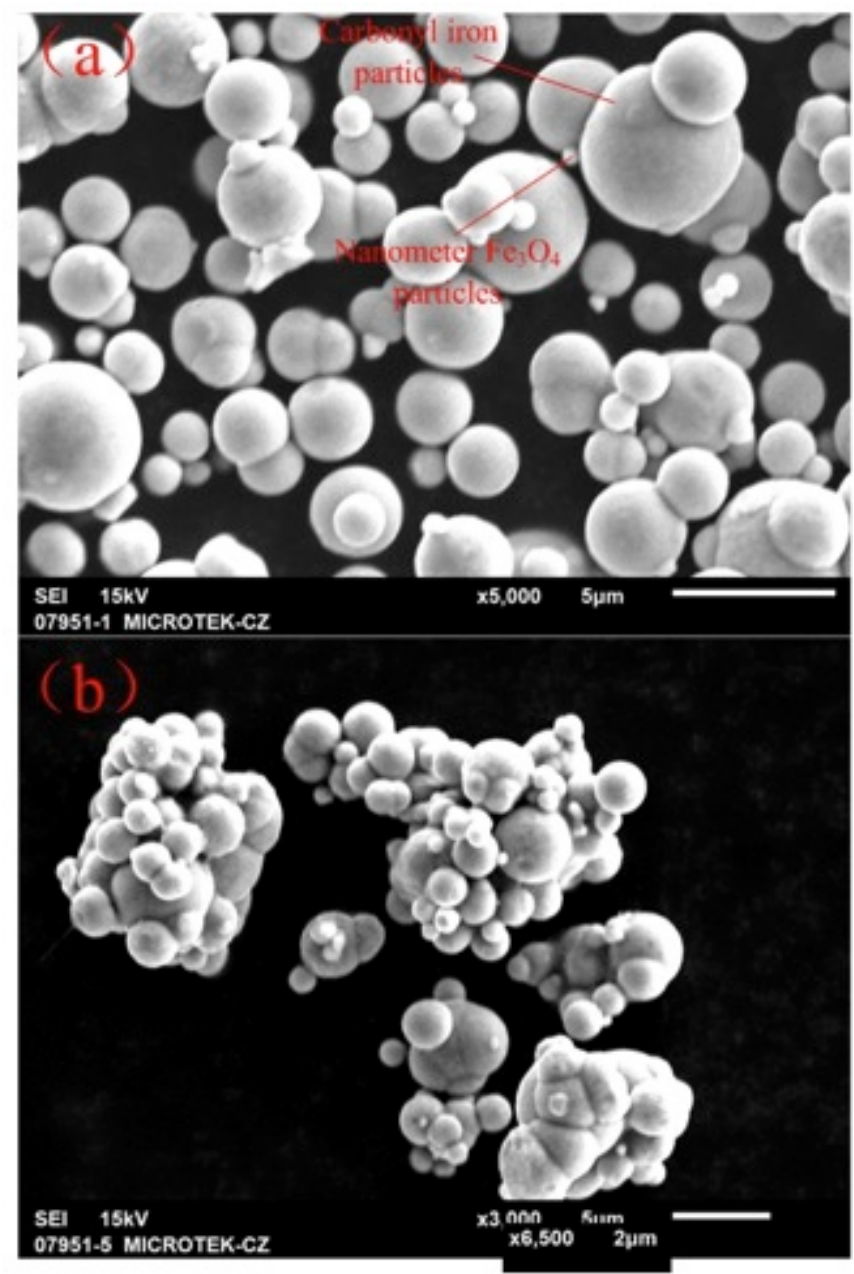

Fig. (3). SEM images of carbonyl iron particles.

More magnetic linkages can be produced with the rising of particles volume fractions and the relative magnetic susceptibility of the system will be better, so the magnetorheological effects become stronger. The yield stress increases as the rising of particles volume fractions in linear only in low volume fractions. The particles would change from unordered state to 3D reticulate structure if two phase suspension liquid has high volume fractions of particles and the liquid would present the curing characteristics and stop flowing with a high apparent viscosity and yield stress as Fig. (3b) shown.

We consider that the magnetic particles are sphere balls with the same diameters $\mathrm{R}$ and under the application of 
applied magnetic field; they are magnetic dipoles which have the same direction as magnetic field [14].

So the magnetic moment of every magnetic particle is shown as:

$m=V M=\frac{4}{3} \pi R^{3} \chi H$

The force situation of the number i particle is shown as:

$F_{m}=\sum_{j \neq i}\left[\frac{3 \mu_{0}}{4 \pi r_{i j}^{5}}\left(m_{i} \bullet m_{j}-5 m_{i r} m_{j r}\right) r_{i j}+\frac{3 \mu_{0}}{4 \pi r_{i j}^{5}}\left(m_{i r} m_{j}+m_{j r} m_{i}\right)\right]$

where $\mu_{0}$ is permeability of vacuum, $r_{i j}$ is the relative position vector between particle $\mathrm{i}$ and $\mathrm{j}, m_{i r}$ and $m_{j r}$ are the component product of $m_{i}$ and $m_{j}$ on the direction of $r_{i j}$. If we define the intersection angle between ligatures of particle $i$ and $\mathrm{j}$ and applied magnetic field as $\theta_{i j}$, the magnetic force expression can be shown as:

$F_{m}=\sum_{j \neq i} \frac{4 \pi \mu_{0} \chi^{2} H^{2} R^{6}}{3 r_{i j}^{4}}\left[\left(1-5 \cos ^{2} \theta_{i j}\right) \hat{r}_{i j}+2 \cos \theta_{i j} \hat{y}\right]$

where $\hat{r}_{i j}$ is the unit vector of $r_{i j}$.

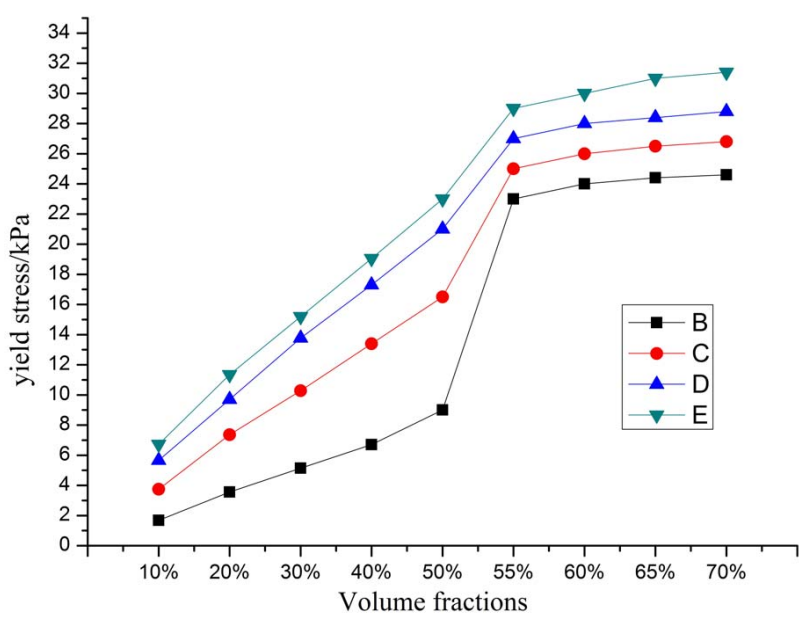

Fig. (4). Saturation phenomenon of particles.

From Fig. (4) we can see that the phenomenon of particles saturation can be divided into three stages. In first stage, the yield stress increases as the rising of particles volume fractions in linear with a low volume fraction $(<45 \%)$ and the effect law is obvious and explicit. In second stage, the yield stress increases abruptly and erratically with a high yield stress. This phenomenon can be explained by the fact that the columnar structure could be composed by more and more magnetic linkages with the increasing volume fraction $(45 \%<55 \%)$. At the last stage, the yield stress keeps a high and stable value because of the $3 \mathrm{D}$ reticulate structure [15]. The MRFs with higher apparent viscosity and yield stress can't be changed drastically with volume fractions even though applied magnetic field. The MRFs with higher volume fractions $(>60 \%)$ are not used widely in engineering because of the bad controllability and narrow rangeability. In addition higher volume fractions are due to high zero field viscosity which has a great influence on transmission efficiency in engineering [16].

As we can see from formula (7), magnetic force increases with the size of magnetic particle increase which leads to a higher yield stress. The requirement of magnetic field intensity to form magnetorheological effects is reduced which means flux linkage can be more likely to form. But the influence of Brownian movement is weakened and the trend of particle sedimentation increase because of the bigger particle size.

\subsection{Saturation Property of Added Nano-Particles}

The incompatible density of particles and carrier liquid are the major reasons to cause poor stability. Many ways were introduced to solve these problems, for example, using chemical modification or encapsulate of inorganic particles with surfactant or polymer, adding non-magnetic particles such as macromolecule protein or nano particles such as Fe$\mathrm{Ni}$ alloy or magnetite in MRFs. In engineering application, nano-particles are usually used in preparation of MRFs because of the bad dispersion stability without improvement measure. The diameter of magnetic-particle is higher than carrier liquid with several orders of magnitudes and there is a larger gap between their densities. For the foregoing reasons, nano-particles as shown in Fig. (3a) are used to perfect the dispersion stability because the diameter and density are between magnetic-particles and carrier liquid. The sedimentation stability is a significant factor affected by the property of MRFs and can be evaluated from sedimentation ratio $\mathrm{S}$ as calculated from: $S=\frac{h}{H} \times 100 \%$.

In the formula, $\mathrm{H}$ and $\mathrm{h}$ express the total length of MRFs and the length of supernatant in the measuring cylinder after sedimentation. The samples with different nano-particles mass fraction were prepared to compare the sedimentation ratio.

From the data of Table 3, it can be seen that the nanoparticles mass fraction has great influence on the sedimentation ratio. As shown, sedimentation ratio of MRFs is reducing from $5.6 \%$ to $2.7 \%$ with the increase of nanometer particles mass fraction from $0 \%$ to $20 \%$. The MRFs would reach the saturation condition when the

Table 3. Sedimentation ratio of MRFs with different mass fraction of nano-particles.

\begin{tabular}{|c|c|c|c|c|c|c|}
\hline Mass Fraction & $\mathbf{0 \%}$ & $\mathbf{5 \%}$ & $\mathbf{7 . 5 \%}$ & $\mathbf{1 0 \%}$ & $\mathbf{1 5 \%}$ & $\mathbf{2 0 \%}$ \\
\hline \hline $\mathbf{4 0}$ hour & $5.6 \%$ & $4.1 \%$ & $3.7 \%$ & $3.4 \%$ & $2.8 \%$ & $2.7 \%$ \\
\hline $\mathbf{6 0}$ hour & $6.7 \%$ & $4.5 \%$ & $4.1 \%$ & $3.7 \%$ & $2.9 \%$. & $2.7 \%$ \\
\hline Yield stress & $\mathbf{1 5 . 2 3} \mathbf{~ k P a}$ & $\mathbf{1 5 . 0 7} \mathrm{kPa}$ & $\mathbf{1 4 . 8 5} \mathbf{k P a}$ & $\mathbf{1 3 . 6 2} \mathbf{k P a}$ & $\mathbf{1 1 . 2 3} \mathbf{k P a}$ & $\mathbf{1 0 . 5 2} \mathbf{k P a}$ \\
\hline
\end{tabular}


nanometer particles mass fraction exceeds $10 \%$. But the influence of nanometer particles is not only reflecting on the sedimentation ratio but also the yield stress. The yield stress would remain unchanged when the nanometer particles mass fraction below 7.5\%, otherwise, a sharp decrease would appear.

\section{CONCLUSION \& FUTURE WORK}

In this paper, saturation phenomena of magnetorheological fluids were analyzed and several experiments were carried out to describe the influences of MRFs parameter design. Through theoretical analysis and experiment research, the influence of magnetic-field saturation, magnetic particles saturation, added nanoparticles saturation on MRFs properties was discussed to provide references for parameter design of MRFs preparation and engineering application.

Future work will focus on the effects of some factors on MRFs such as the size of magnetic particles, temperature field, etc. Furthermore, the improvement of magnetorheological fluids transmission devices is also an important research for the authors.

\section{ABBREVIATIONS}

$$
\begin{aligned}
& \text { MRFs }=\text { Magnetorheological fluids } \\
& \text { SEM }=\text { Scanning electron microscopy }
\end{aligned}
$$

\section{CONFILCT OF INTEREST}

The authors confirm that this article content has no conflict of interest.

\section{ACKNOWLEDGEMENTS}

The support of National Natural Science Foundation of China (No. 51475454), Xuyi Mine Equipment and Materials R\&D Center Innovation Fund Project (No. CXJJ201306), Open Foundation of the State Key Laboratory of Fluid Power Transmission and Control (No. GZKF-201316) and A Project Funded by the Priority Academic Program Development of Jiangsu Higher Education Institutions in carrying out this research are gratefully acknowledged.

\section{REFERENCES}

[1] R. Hiegeister, W. Andra, N. Buske, R. Hergt, I. Hilger, U. Richter, and W. Kaiser, "Application of magnetite ferro fluids for hyperthermia", Journal of Magnetism and Magnetic Materials, vol. 201, pp. 420-422, 1999.

[2] S. Kang, and Y. K. Suh: "Direct simulation of flows with suspended paramagnetic particles using one-stage smoothed profile method", Journal of Fluids and Structures, vol. 27, no. 2, pp. 266282, 2011.

[3] G. Bossis, and O. Volkova: Magnetorheology Fluids, Structures and Rheology, Springer-Verlag: Berlin Heidelberg, vol. 594, pp. 202-230, 2002.

[4] K. Nakatsuka, B. Jeyadevan, S. Neveu, and H. Koganezawa: "The magnetic fluid for heat transfer applications", Journal of Magnetism and Magnetic Materials, vol. 252, pp. 360-362, 2002.

[5] M. Aoshima, and A. Satoh: "Two-dimensional Monte Carlo simulations of a colloidal dispersion composed of rod-like ferromagnetic particles in the absence of an applied magnetic field", Journal of Colloid and Interface Science, vol.293, pp. 7787, 2006.

[6] X. Yimin, Y. Meng, and L. Qiang, "Lattice Boltzmann simulation of ferrofluid structure", Journal of Engineering Thermophysics, vol. 26, no.2, pp. 301-303, 2005.

[7] A. Chaudhuri, G. Wang, N. M. Wereley, V. Tasovksi, and R. Radhakrishnan, "Substitution of micron by nanometer scale powders in magnetorheological fluids", International Journal of Modern Physics, vol. 19, pp. 1374-1380, 2005.

[8] J. M. Ginder, and L. C. Davis: "Shear stresses in magnetorheological fluids: role of magnetic saturation", Applied Physics Letters, vol. 65 , no. 26 , pp. 3410-3412, 1994.

[9] W. A. Bullogh, Proceedings of the $5^{\text {th }}$ International Conference on ER Fluids, MR Suspensions and Associated Technology, Singapore: World Scientific, 1996, pp. 738-746.

[10] G. Bossis, E. Lemaire, and O. Volkova: "Yield stress in magnetorheological and electrorheological fluids: a comparison between microscopic and macroscopic structural models", Journal of Rheology, vol. 41, no.3, pp. 687-704, 1997.

[11] E. Lemaire, A. Meunier, and G. Bossis: "Influence of the particle size on the rheology of magnetorheological fluids", Journal of Rheology, vol.39, no.5, pp. 1011-1020, 1995.

[12] S. Yuan, H. Ren, and Y. Luo, "The research and application of MRF magnetic circuit design", The Open Mechanical Engineering Journal, vol. 9, pp. 241-245, 2015.

[13] M. Nakano, and K. Koyama, Proceedings of the $6^{\text {th }}$ International Conference on ER fluids, MR Suspensions and their Applications, Yonezawa: World Scientific pp. 494-50, 1998.

[14] J. Ren, and X. Guo, "Numerical simulation of middle thick plate in the u-shaped bending spring back and the change of thickness", The Open Mechanical Engineering Journal, vol. 8, pp. 648-654, 2014.

[15] Z. Niu, W. Ma, and J. Li, "High-strength laminated copper matrix nanocomposites developed from a single-walled carbon nanotube film with continuous reticulate architecture", Advanced Functional Materials vol.22, no.24, pp. 5209-5215, 2012.

[16] S. Zheng, X. Wang, and R. Zheng: "Computation of viscous flow inside a double-channel centrifugal pump", The Open Mechanical Engineering Journal, vol. 8, pp. 613-618, 2014. 